\title{
ORIGINAL ARTICLE \\ rTMS of the prefrontal cortex has analgesic effects on neuropathic pain in subjects with spinal cord injury
}

\author{
R Nardone ${ }^{1,2,3}$, Y Höller ${ }^{1,3}$, PB Langthaler ${ }^{1,3,4}$, P Lochner $^{2}$, S Golaszewski ${ }^{1,3}$, K Schwenker ${ }^{1,3}$, F Brigo ${ }^{2,5}$ \\ and $\mathrm{E}$ Trinka ${ }^{1,3}$
}

Study design: Repetitive transcranial magnetic stimulation study.

Objectives: The analgesic effects of repetitive transcranial magnetic stimulation (rTMS) in chronic pain have been the focus of several studies. In particular, rTMS of the premotor cortex/dorsolateral prefrontal cortex (PMC/DLPFC) changes pain perception in healthy subjects and has analgesic effects in acute postoperative pain, as well as in fibromyalgia patients. However, its effect on neuropathic pain in patients with traumatic spinal cord injury (SCI) has not been assessed.

Setting: Merano (Italy) and Salzburg (Austria).

Methods: In this study, we performed PMC/DLPFC rTMS in subjects with $\mathrm{SCl}$ and neuropathic pain. Twelve subjects with chronic cervical or thoracic SCl were randomized to receive 1250 pulses at $10 \mathrm{~Hz}$ rTMS $(n=6)$ or sham rTMS $(n=6)$ treatment for 10 sessions over 2 weeks. The visual analog scale, the sensory and affective pain rating indices of the McGill Pain Questionnaire (MPQ), the Hamilton Depression Rating Scale and the Hamilton Anxiety Rating Scale were used to assed pain and mood at baseline (TO), 1 day after the first week of treatment (T1), 1 day (T2), 1 week (T3) and 1 month (T4) after the last intervention.

Results: Subjects who received active rTMS had a statistically significant reduction in pain symptoms in comparison with their baseline pain, whereas sham rTMS participants had a non-significant change in daily pain from their baseline pain.

Conclusion: The findings of this preliminary study in a small patient sample suggest that rTMS of the PMC/DLPFC may be effective in relieving neuropathic pain in $\mathrm{SCl}$ patients.

Spinal Cord (2017) 55, 20-25; doi:10.1038/sc.2016.87; published online 31 May 2016

\section{INTRODUCTION}

Human brain imaging studies as well as animal model studies have emphasized the role of brain plasticity and cortical reorganization in chronic pain. If delivered repetitively, transcranial magnetic stimulation (TMS) can also modulate cortical excitability and induce long-lasting neuroplastic changes. ${ }^{1}$ Stimulating the human cortex using repetitive TMS (rTMS) temporarily reduces clinical and experimental pain. However, it is unclear which cortical targets are the most effective, and the mechanisms of central pain relief remain poorly understood. ${ }^{2}$ To date, most studies have targeted the primary motor cortex (M1), mainly contralateral to the pain side, ${ }^{3}$ whereas fewer studies reported analgesic effects after stimulation of other cortical areas, such as the prefrontal cortex (PFC)/dorsolateral PFC (DLPFC). ${ }^{4-8}$ Also for managing neuropathic pain in subjects with spinal cord injury (SCI), M1 has been a popular target, ${ }^{9-14}$ but it is unclear whether M1 is the only effective cortical target. To date, no studies have thoroughly investigated the effects of stimulation of other cortical targets (that is, PFC, parietal areas, supplementary motor area, primary or secondary somatosensory cortex) on neuropathic pain in SCI patients. In particular, stimulation of the
DLPFC remains to be studied, according to the proven efficacy of this target in depression and the well-known relation between chronic pain and depression.

The analgesic mechanisms of rTMS to the PFC/DLPFC seem not to be the same as those of M1 stimulation ${ }^{15}$ and may include top-down modulation of the medial spinal thalamic system with a preferential effect in the affective and evaluative aspects of pain. ${ }^{16-18}$ rTMS for M1 and DLPFC promotes differences in the central processing of heat and cold stimuli, and acts on neuronal networks that are involved in cold pain processing. ${ }^{15,19}$

To the best of our knowledge, there are no studies of high-frequency rTMS over the left DLPFC for SCI, despite the body of evidence to support the antidepressant effect of high-frequency rTMS of the left DLPFC. ${ }^{2}$ As the stimulation of prefrontal regions has been demonstrated to be effective in the treatment of depression, and there is a close relationship between depression and chronic pain, ${ }^{20,21}$ the potential value of this target should be better evaluated. rTMS may modify overlapping mood and pain regulation circuitry, particularly with stimulation of left DLPFC.

${ }^{1}$ Department of Neurology, Christian Doppler Klinik, Paracelsus Medical University and Center for Cognitive Neuroscience, Salzburg, Austria; ${ }^{2}$ Department of Neurology, Franz Tappeiner Hospital, Merano, Italy; ${ }^{3}$ Spinal Cord Injury and Tissue Regeneration Center, Paracelsus Medical University, Salzburg, Austria; ${ }^{4}$ Department of Mathematics, Paris Lodron University of Salzburg, Salzburg, Austria and ${ }^{5}$ Department of Neurological and Movement Sciences, University of Verona, Verona, Italy

Correspondence: Dr R Nardone, Department of Neurology, Franz Tappeiner Hospital, Via Rossini, 5, Merano 39012, Italy.

E-mail: raffaele.nardone@asbmeran-o.it

Received 19 September 2015; revised 26 November 2015; accepted 30 November 2015; published online 31 May 2016 
We hypothesized that subjects with SCI might experience a clinically significant reduction in daily pain after stimulation of the left PFC/DLPFC.

\section{MATERIALS AND METHODS}

\section{Patients}

Twelve subjects with cervical or thoracic SCI were enrolled in the study. The subjects were randomly assigned into two groups: six subjects received active rTMS and six subjects received sham rTMS. Clinical and demographic features of the subjects are shown in Table 1.

All of the subjects had chronic pain with exclusively or predominantly neuropathic character, fulfilling the International Association for the Study of Pain criteria for central neuropathic pain, ${ }^{22}$ at and/or below the level of injury.

The pain intensity was at least $4 / 10$ in the visual analog scale ${ }^{23}$ despite rehabilitation and pharmacological treatment.

Other inclusion criteria were recordable motor-evoked potential with an amplitude of at least $0.1 \mathrm{mV}$ in relaxed first dorsal interosseous after TMS, and the ability to give informed consent and comprehend instructions.

Exclusion criteria were significant medical or psychiatric illnesses (except for reactive depression), history of epilepsy or seizures, pregnancy or metal objects in the head.

All the subjects were previously treated with antidepressant, anticonvulsants and analgesics for a minimum period of 6 months, in accordance with the International Association for the Study of Pain guidelines for neuropathic pain treatment. $^{24}$

All the subjects provided informed consent before participation in this study, which was performed according to the Declaration of Helsinki and approved by the local Ethics Committee.

\section{Transcranial magnetic stimulation}

The subjects were seated in a comfortable reclining chair and asked to keep their hands as relaxed as possible. Magnetic stimulation was applied with a MagStim Super Rapid magnetic stimulator (Magstim Company, Whitland, UK), using a figure-8-shaped coil oriented at a tangent to the scalp, with the main phase of the induced current in the anterior-posterior direction, and fixed to an arm that could be adjusted in three dimensions. ${ }^{25}$ The subjects were fitted with earplugs during rTMS. Sham stimulation was carried out with a sham coil of identical size color and shape emitting a sound similar to that emitted by the

Table 1 Clinical and demographic characteristics of the subjects with spinal cord injury

\begin{tabular}{|c|c|c|c|c|c|c|}
\hline Patients & $A(y)$ & $G$ & Etiology & Time since $\mathrm{SCl}(y)$ & Level/ASIA & Medications \\
\hline \multicolumn{7}{|l|}{ a-rTMS } \\
\hline 1 & 56 & $M$ & Disc prolaps & 19 & $C 6 / D$ & SRT, PGB \\
\hline 2 & 26 & $\mathrm{~F}$ & Fracture & 6 & $\mathrm{C} 7 / \mathrm{B}$ & AMT, GBP \\
\hline 3 & 50 & M & Fracture & 7 & $C 5 / D$ & AMT, PGB \\
\hline 4 & 30 & M & Fracture & 10 & $\mathrm{C} 7 / \mathrm{C}$ & VLF, GBP \\
\hline 5 & 46 & M & Fracture & 13 & $\mathrm{~T} 4 / \mathrm{A}$ & $\mathrm{CBZ}$ \\
\hline 6 & 54 & $\mathrm{~F}$ & Fracture & 4 & T8/B & AMT \\
\hline \multicolumn{7}{|l|}{$s-r T M S$} \\
\hline 1 & 26 & $M$ & Fracture & 6 & $\mathrm{C} 7 / \mathrm{B}$ & AMT \\
\hline 2 & 44 & $M$ & Fracture & 15 & $C 7 / D$ & SRT, PGB \\
\hline 3 & 47 & $\mathrm{~F}$ & Fracture & 12 & C6/B & VLF, PGB \\
\hline 4 & 52 & $M$ & Fracture & 10 & $\mathrm{C} 7 / \mathrm{B}$ & AMT, GBP \\
\hline 5 & 24 & M & Fracture & 4 & $\mathrm{~T} 10 / \mathrm{C}$ & AMT \\
\hline 6 & 62 & M & Fracture & 17 & $\mathrm{~T} 6 / \mathrm{A}$ & VLF \\
\hline
\end{tabular}

Abbreviations: A, age; a-rTMS, active repetitive transcranial magnetic stimulation; AMT, amitriptylin; ASIA, American Spinal Cord Injury Association Impairment Scale; CBZ, carbamazepine; F, female; G, gender; GBP, gabapentin; M, male; PGB, pregabalin; SCl, spinal cord injury; S-rTMS, sham repetitive transcranial magnetic stimulation; SRT, sertraline; VLF, venlafaxine; $y$, years. active coil. Resting motor threshold was determined by single-pulse stimulation of the left M1. Motor-evoked potentials were recorded for the right first interosseous muscle (first dorsal interosseous), with an electromyogram amplifier module and surface electrodes. Resting motor threshold was defined as the lowest intensity required to elicit a motor-evoked potential of at least $50 \mathrm{mV}$ in $50 \%$ of the successive trials. ${ }^{26}$

As the left PFC was the cortical target, a mark was made $6 \mathrm{~cm}$ anterior to the M1 target. This may be reasonably accurate with respect to locating Brodman's area 9, given that standard ' 5 -cm rule' compared with image-guided TMS coil positioning for DLPFC can be $\sim 1$ or $2 \mathrm{~cm}$ posterior to target location at least $30 \%$ of the time. ${ }^{27,28}$ We opted not to employ neuronavigation procedures also in order to maximize the potential feasibility of the protocol for widespread use. During active and sham stimulation, the TMS coil was aligned in a postero-anterior orientation, $6 \mathrm{~cm}$ from the area that produced right first dorsal interosseous muscle movement for resting motor threshold testing. The participants were subjected to daily rTMS sessions (five per week) for 2 weeks. Each session consisted of 25 series of 5-s pulses of $10 \mathrm{~Hz}$ with an interval of $25 \mathrm{~s}$ between each train of rTMS, totaling 1250 pulses per session. The intensity of stimulation was $120 \%$ of the resting motor threshold. All the rTMS sessions were performed by two trained neurophysiologists who were blinded to the clinical evaluation and had no role in data collection.

\section{Clinical assessment}

Pain and mood assessments were performed at baseline (T0), 1 day after the first week of treatment (T1), 1 day (T2), 1 week (T3) and 1 month (T4) after the last intervention.

Pain was assessed by an investigator blinded to the type of rTMS subjects were receiving.

The primary outcome variables were as follows: (1) a generic unidimensional pain questionnaire and the visual analog scale $(0-10)$, a continuous scale comprising a horizontal line, usually $10 \mathrm{~cm}$ in length, anchored by two verbal descriptors, one for each symptom extreme; (2) a generic multidimensional pain questionnaire, the sensory pain rating index (score 0-42) and the affective pain rating index (score $0-14$ ) of the $\mathrm{MPQ}^{29}$ that describe pain characteristics in two dimensions of pain, sensory qualities (word groups 1-10 and 17-19; described in terms of temporal, spatial, pressure, thermal and other properties) and affective qualities (word groups 11-15, 20; described in terms of tension, fear and autonomic properties), respectively.

Secondary clinical outcomes were the effects on mood symptoms; the multiple item questionnaires Hamilton Rating Scale for Depression (HAM-D, ${ }^{30}$ mild: score 8-13; moderate: 14-18; and severe: $>19$ ) and the Hamilton Anxiety Rating Scale (HAM-A, ${ }^{31}$ mild: $14-17$; moderate: $18-24$; and severe: $\geqslant 25$ ) were used.

\section{Statistical analysis}

Statistics were carried out using the software environment R (R Core Team, Vienna, Austria, (2013)), including the package nparLD, with fully nonparametric analysis of variance-type testing, for which a rank-based finite sample approximation based on quantiles of the F distribution is derived. ${ }^{32}$ Thus, we included the between-factor group (active rTMS vs sham rTMS) and the within-factor time (T0, T1, T2, T3 and T4).

As the samples are small, we avoided multiple testing of each single pain score and instead built a sum score of visual analog scale and the two sub-scales of MPQ (sensory pain rating index and affective pain rating index). For this, we normalized the MPQ sub-scales to have values between 0 and 10, by dividing them through the maximal achievable value (that is, 33 and 12) and then multiplying them by 10 . This resulted in three variables ranging from 0 to 10 (visual analog scale, MPQ1Adjusted and MPQ2Adjusted). We then defined the sum score to be the sum of these three variables. By normalizing before summation, we made sure that each variable contributed to the sum score equally. Without normalization, for example, the MPQ subscale sensory pain rating index would have skewed the sum score by contributing a potentially larger value than each of the other two could, even though not being deemed more important for clinical outcome. For illustration purposes, also the scores of HAM-D and HAM-A were normalized to values between 0 and 10 on the 
plots. The latter two variables were considered secondary and were analyzed using a Bonferroni-Holm multiple testing correction.

Post hoc tests were applied in case of a significant main effect in the within-subject factor time. For this, we also used nparLD and corrected the $P$-values according to Holm-Bonferroni.

As a measure of effect, we used the relative treatment effect (RTE), which can be interpreted as follows: a RTE of 0.3 for a subgroup of our sample means that the probability of a randomly drawn subject from the whole sample having a lower score than a randomly drawn person of the subgroup is estimated to be $30 \%$. Thus, the RTE is a number between 0 and 1 . A RTE of 0.5 means no effect (that is, a randomly drawn person from the whole sample has a $50-50$ chance of achieving a lower value than a randomly drawn person from the subgroup). A RTE $<0.5$ means a tendency for subjects in a subgroup to score lower than a random subject from the whole sample. A RTE $>0.5$ means a tendency for subjects in a subgroup to score at least as high as a randomly drawn subject from the whole sample.

\section{RESULTS}

All the subjects tolerated the study well. Two subjects undergoing active rTMS reported slightly unconfortable twiching of facial muscles during rTMS.

Table 2 shows the results of the functional outcome scores at the different time points after rTMS application.

The analysis of variance-type test for the sum score of pain revealed a significant main effect for group $\left(\mathrm{F}_{(1, \infty)}=1.71 ; P=.02\right.$; $\mathrm{RTE}=0.41$ for treatment), a significant main effect for time $\left(\mathrm{F}_{(2.27, \infty)}=26.55\right.$; $P<.001$; RTEs $=0.61,0.38,0.36,0.55$ and 0.60 for T0, T1, T2, T3 and $\mathrm{T} 4$, respectively) and a significant interaction between group and time $\left(\mathrm{F}_{(1.27, \infty)}=20.16 ; P<.001\right.$; RTEs for treatment: 0.60, 0.19, 0.15, 0.51 and 0.59 for T0, T1, T2, T3 and T4, respectively; RTEs for sham: 0.62 , $0.58,0.58,0.59$ and 0.61 for T0, T1, T2, T3 and T4, respectively). The RTE of 0.41 for the treatment is $<0.5$, thus indicating that treatment resulted in a lower score. The RTEs for time indicate that at T0 and T1 subjects tended to have a much lower score than at the other time points. When looking at the RTEs for the interaction between treatment and time one can see that this is due to subjects in the treatment group scoring much lower at those time points, whereas subjects in the sham group did not differ much in their scores over time. Figure 1 illustrates the results for the three normalized pain-scores individually, as well as the sum score. During treatment, the pain-scores decreased in the treatment group but not in the sham group. According to post hoc tests, a significant $\left(\mathrm{F}_{(1, \infty)}=14.80\right.$; $P<.001$; RTE $=0.28$ for treatment) difference between groups was reached after the 2 weeks of treatment. In the follow-up period, the pain rebounded to pre-treatment scores in the treatment group.

The analysis of variance-type test for HAM-D revealed a significant main effect for time $\left(\mathrm{F}_{(2.19, \infty)}=13.34 ; P<.001\right.$; RTEs $=0.57,0.52$, $0.40,0.46$ and 0.56 for T0, T1, T2, T3 and T4, respectively), indicating a worsening of depressive symptoms between $\mathrm{T} 1$ and $\mathrm{T} 2$, with a subsequent return to previous values, and a significant interaction between group and time $\left(\mathrm{F}_{(2.19, \infty)}=10.35 ; P<.001\right.$; RTEs for treatment: $0.67,0.62,0.36,0.49$ and 0.67 for T0, T1, T2, T3 and T4, respectively; RTEs for sham: $0.47,0.42,0.43,0.43$ and 0.45 for T0, $\mathrm{T} 1, \mathrm{~T} 2, \mathrm{~T} 3$ and $\mathrm{T} 4$, respectively), indicating that the main effect for time can be explained by subjects in the treatment group experiencing a large drop in depressive symptoms between $\mathrm{T} 1$ and $\mathrm{T} 2$, whereas

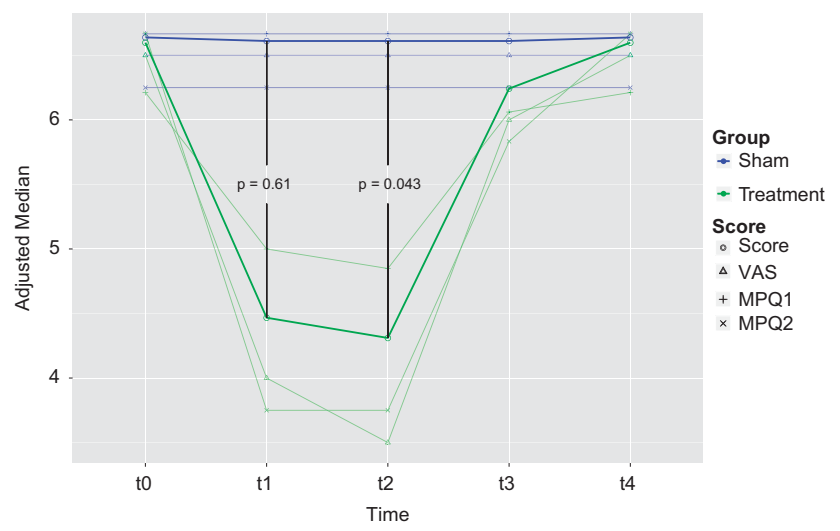

Figure 1 Means of the different pain scales as well as the sum score by group and time. All scores were normalized to a range between 0 and 10 . Error bars represent one sample s.d.

Table 2 Primary and secondary functional outcome scores: baseline values and changes after rTMS application

\begin{tabular}{|c|c|c|c|c|}
\hline VAS & $M P Q S-P R I$ & $M P Q A-P R I$ & $H A M-D$ & $H A M-A$ \\
\hline \multicolumn{5}{|l|}{ a-rTMS } \\
\hline T0 T1 T2 T3 T4 & T0 T1 T2 T3 T4 & T0 T1 T2 T3 T4 & T0 T1 T2 T3 T4 & T0 T1 T2 T3 T4 \\
\hline 64466 & 2015151920 & 75577 & 1414111314 & 10991010 \\
\hline 85578 & 2419192324 & 84478 & 1715121317 & 1514131414 \\
\hline 96588 & 3024222929 & 11761010 & 2220151720 & 2020191920 \\
\hline 43344 & 1513121515 & 53355 & 1213111213 & 1414141414 \\
\hline 64366 & 2017171921 & 96699 & 2019161819 & 1313131313 \\
\hline 74367 & 2116152121 & 84378 & 1413101314 & 1514151515 \\
\hline \multicolumn{5}{|l|}{ s-rTMS } \\
\hline 55555 & 1515151515 & 77777 & 1313131313 & 1313131313 \\
\hline 77767 & 2524242425 & 98888 & 1513141414 & 1211111111 \\
\hline 98999 & 3020303030 & 1110101111 & 2020191920 & 1716161617 \\
\hline 88888 & 2424232424 & 77767 & 1010101010 & 1313131213 \\
\hline 66666 & 2020202020 & 55555 & 88888 & 109101010 \\
\hline 66666 & 2020202020 & 88888 & 1515151515 & 1212121212 \\
\hline
\end{tabular}

Abbreviations: a-rTMS, active repetitive transcranial magnetic stimulation; A-PRI, affective pain rating index; HAM-A, Hamilton Anxiety Rating Scale; HAM-D, Hamilton Rating Scale for Depression; MPQ, McGill Pain Questionnaire; s-rTMS, sham repetitive transcranial magnetic stimulation; S-PRI, sensory pain rating index; VAS, visual analog scale; TO, baselinel; T1, 1 day after the first week of treatment; T2, 1 day after the last intervention; T3, 1 week after the last intervention; T4, 1 month after the last intervention. 


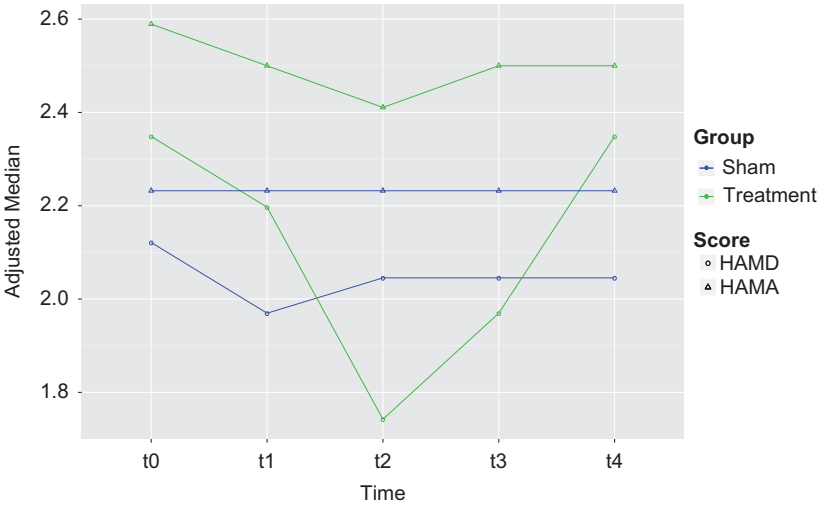

Figure 2 Means of the HAM-D and HAM-A scores by group and time. The scores were normalized to a range between 0 and 10 . Error bars represent one sample s.d.

scores stayed similar for the sham group. The sham group having consistently lower RTEs than 0.5 can be explained by this group having smaller scores on the HAM-D at baseline. The main effect for group $\left(\mathrm{F}_{(1, \infty)}=0.54 ; P=.046 ; \mathrm{RTE}=0.56\right.$ for treatment $)$ was not significant after correcting for multiple comparisons.

The analysis of variance-type test for HAM-A revealed no significant effects.

Figure 2 illustrates the results for HAM-D and HAM-A scores. Similar to the pain-scores, anxiety decreased during treatment but reached pre-treatment levels in the follow-up period. There were no sham effects on anxiety, and neither active nor sham rTMS significantly affected depression.

\section{DISCUSSION}

We found that 10 sessions of high-frequency left prefrontal rTMS resulted in statistically significant reductions in daily pain over time in a group of TMS-naive outpatients. In contrast to our results, analgesic effect of rTMS was found to be not superior to placebo on intractable neuropathic pain in SCI in another recent study. ${ }^{33}$ This discrepancy can be explained by the different target, as the rTMS was applied over the vertex.

rTMS applications over the M1 also failed to demonstrate analgesic effects. Lefaucheur et al. ${ }^{9}$ applied high-frequency rTMS over the M1 in 60 patients with drug-resistant neurogenic pain, including 12 subject with SCI. The effects were significantly influenced by pain origin and site, and the results were worse in subjects with spinal cord and brainstem lesions. Defrin et al. ${ }^{11}$ found similar improvement in pain by real and sham rTMS after 10 treatment sessions in 11 subjects with central pain of spinal origin. Kang et al. ${ }^{12}$ reported no reduction in average pain in 11 subjects with chronic complete and incomplete SCI after a 5-day treatment.

The findings of our study are consistent with several functional neuroimaging studies showing that the prefrontal cortex is critically involved in pain modulation ${ }^{18,34,35}$ and also in patients with neuropathic pain following SCI. ${ }^{36,37}$ Atrophy of the DLPFC has been consistently found in previous brain morphometric studies of various chronic pain conditions, ${ }^{38-40}$ and previous neuroimaging studies suggested that the DLPFC has a central role in top-down pain processing. A positron emission tomographic imaging study has shown that, in painful thermal stimulation of normal and capsaicin-treated skin in healthy volunteers, the activity of the DLPFC was negatively correlated with the intensity of perceived pain and unpleasantness; moreover, an inhibition of the neuronal coupling between the midbrain and thalamus was observed during high activity of the left DLPFC. ${ }^{18}$ On the other hand, experimental studies have revealed that stimulation of the PFC can minimize noxious behavior and reduces the activity of the midbrain areas involved in the processing of pain. ${ }^{16,17}$

More specifically, it has been recently observed in subjects with chronic neuropathic pain after SCI that metabolism and the gray matter volume decrease in the left DLPFC compared with healthy controls, as well as hypometabolism in the medial PFC, and gray matter volume loss in bilateral anterior insulae and subgenual anterior cingulate cortices. ${ }^{41}$ These brain regions are generally known to participate in pain modulation by affective and cognitive processes.

Pain modulation circuitry may involve PFC, anterior cingulate cortex, periaqueductal gray and ventral medial medulla. ${ }^{42}$ It has been hypothesized that chronic repetitive stimulation of the PFC initiates a cascade of events in the PFC and in connected limbic regions. ${ }^{28}$ Prefrontal TMS sends information to important mood-regulating regions including the cingulate gyrus, orbitofrontal cortex, insula and hippocampus and may induce dopamine release in the caudate nucleus. ${ }^{28,43}$

High-frequency rTMS in the DLPFC might activate rostral anterior cingulate activity and pain control circuitry to facilitate the placebo response by endogenous opioid release. ${ }^{44}$ In fact, rTMS of the left DLPFC exerts strong modulation of the brain activity in the frontal-cingulate circuit involved in mood control ${ }^{43,45}$ and also improved pain in patients with major depression regardless of its antidepressant action, suggesting a primary antalgic action. ${ }^{46}$

A recent study identified that high-frequency rTMS of the left DLPFC leads to reduced allodynia pain ratings and further found that this analgesia was associated with increased activity in the DLPFC and decreased activity in the anterior cingulate cortex, thalamus, midbrain and medulla. Roles of the DLPFC in attentional processes ${ }^{47}$ and executive function ${ }^{48}$ are also believed to be related to cognitive modulation of pain processes.

Interestingly, experimental pain studies have shown that rTMS of the DLPFC can suppress the perceived control on the emotional dimension of the pain experience. ${ }^{5}$

rTMS of left DLPFC may thus modify overlapping mood and pain regulation circuitry. The antidepressant and analgesic effects might share common mechanisms. In the present study, SCI patients experienced a clinically significant reduction in their daily neuropathic pain even if rTMS was administered in a manner different from rTMS antidepressant protocols.

Nevertheless, we found a decrease in depression at the end of the 2 weeks of active rTMS versus sham treatment. Therefore, pain reduction occurred before changes in mood, similar to what has been reported after rTMS in subjects with fibromyalgia. ${ }^{8}$ As both depression and anxiety are comorbid features in subjects with SCI, other studies are necessary to verify this important issue.

In subjects with central post-stroke pain, no significant analgesic effects were found after rTMS of DLPFC. ${ }^{49}$ Different pain syndromes may respond differently to rTMS protocols, depending on the use of different targets and sides of stimulation. The absence of analgesic effects of premotor cortex/DLPFC rTMS in central post-stroke pain may suggest a different pain generation and modulation mechanism specific to this patient population.

The method of coil location utilized was the same as used for the treatment of depression ${ }^{50-52}$ but was not neuronavigated. There is evidence that the neuronavigation-guided targeting of DLPFC offers greater precision in the location of the target ${ }^{53}$ and DLPFC can be 
almost $2 \mathrm{~cm}$ more anterior ${ }^{26}$ than the point found by the $5-\mathrm{cm}$ rule. This is why we chose to name it premotor cortex/DLPFC. ${ }^{15,54}$

Also, scalp sensations are clearly different between active and sham rTMS sessions. This issue concerns rTMS trials in general, and it has been neglected in the vast majority of studies available so far. However, because the patients were rTMS naive and the study had a parallel design, it is likely that the influence of higher discomfort during rTMS in the active group had a minor role in the different findings of the present study.

Even if care must be taken before drawing conclusions from reports with a small sample size, this is the first study showing that multiple sessions of rTMS on left premotor cortex/DLPFC are effective in relieving chronic pain in SCI patients. These results confirm that rTMS may represent a promising therapeutic tool in chronic pain, and attest to an actual biological effect of the active rTMS applied on this target. rTMS has the main advantage that is non-invasive and can be applied to any patient with pain who are resistant to the pharmacological treatment. However, its clinical effect is short lasting, and rTMS cannot be considered as a long-term treatment method. It should be addressed in further studies whether the short-lasting pain reduction found in this preliminary report may have therapeutic significance. Different rTMS protocols in combination with pharmacological approaches should also be evaluated. Overall, a personalized approach with respect to affective emotional components of pain might enhance the analgesic effect of rTMS in individuals with neuropathic pain.

\section{CONFLICT OF INTEREST}

The authors declare no conflict of interest.

\section{DATA ARCHIVING}

There were no data to deposit.

1 Hallett M. Transcranial magnetic stimulation: a primer. Neuron 2007; 55: 187-199.

2 Klein MM, Treister R, Raij T, Pascual-Leone A, Park L, Nurmikko T et al. Transcranial magnetic stimulation of the brain: guidelines for pain treatment research. Pain 2015; 156: 1601-1614.

3 Lefaucheur JP, André-Obadia N, Antal A, Ayache SS, Baeken C, Benninger DH et al. Evidence-based guidelines on the therapeutic use of repetitive transcranial magnetic stimulation (rTMS). Clin Neurophysiol 2014; 125: 2150-2206.

4 Borckardt JJ, Reeves ST, Weinstein M, Smith AR, Shelley N, Kozel FA et al. Significant analgesic effects of one session of postoperative left prefrontal cortex repetitive transcranial magnetic stimulation: a replication study. Brain Stimul 2008; 1: 122-127

5 Borckardt JJ, Reeves ST, Frohman H, Madan A, Jensen MP, Patterson D et al. Fast left prefrontal rTMS acutely suppresses analgesic effects of perceived controllability on the emotional component of pain experience. Pain 2011; 152: 182-187.

6 Brighina F, De Tommaso M, Giglia F, Scalia S, Cosentino G, Puma A et al. Modulation of pain perception by transcranial magnetic stimulation of left prefrontal cortex. J Headache Pain 2011; 12: 185-191.

7 Fierro B, De Tommaso M, Giglia, Giglia G, Palermo A, Brighina F. Repetitive transcranial magnetic stimulation (rTMS) of the dorsolateral prefrontal cortex (DLPFC) during capsaicin-induced pain: modulatory effects on motor cortex excitability. Exp Brain Res 2010; 203: 31-38.

8 Short EB, Borckardt JJ, Anderson BS, Frohman H, Beam W, Reeves ST et al. Ten sessions of adjunctive left prefrontal rTMS significantly reduces fibromyalgia pain: a randomized, controlled pilot study. Pain 2011; 152: 2477-2484.

9 Lefaucheur JP, Drouot X, Menard-Lefaucheur I, Zerah F, Bendib B, Cesaro P et al. Neurogenic pain relief by repetitive transcranial magnetic cortical stimulation depends on the origin and the site of pain. J Neurol Neurosurg Psychiatry 2004; 75: 612-657.

10 Lefaucheur JP, de Andrade DC. Intraoperative neurophysiologic mapping of the central cortical region for epidural electrode placement in the treatment of neuropathic pain by motor cortex stimulation. Brain Stimul 2009; 2: 138-148.

11 Defrin R, Grunhaus L, Zamir D, Zeilig G. The effect of a series of repetitive transcranial magnetic stimulations of the motor cortex on central pain after spinal cord injury. Arch Phys Med Rehabil 2007; 88: 1574-1580.
12 Kang BS, Shin HI, Bang MS. Effect of repetitive transcranial magnetic stimulation over the hand motor cortical area on central pain after spinal cord injury. Arch Phys Med Rehabil 2009; 90: 1766-1771.

13 Jetté F, Côté I, Meziane HB, Mercier C. Effect of single-session repetitive transcranial magnetic stimulation applied over the hand versus leg motor area on pain after spinal cord injury. Neurorehabil Neural Repair 2013; 27: 636-643.

14 Tazoe T, Perez MA. Effects of repetitive transcranial magnetic stimulation on recovery of function after spinal cord injury. Arch Phys Med Rehabil 2015; 96: S145-S155.

15 de Andrade DC, Mhalla A, Adam F, Texeira MJ, Bouhassira D. Neuropharmacological basis of rTMS-induced analgesia: the role of endogenous opioids. Pain 2014; 155: 598-605.

16 Hardy SG. Analgesia elicited by prefrontal stimulation. Brain Res 1985; 339: 281-284.

17 Hardy SG, Haigler HJ. Prefrontal influences upon the midbrain: a possible route for pain modulation. Brain Res 1985; 339: 285-293.

18 Lorenz J, Minoshima S, Casey KL. Keeping pain out of mind: the role of the dorsolateral prefrontal cortex in pain modulation. Brain 2003; 126: 1079-1091.

19 Nahmias F, Debes C, de Andrade DC, Mhalla A, Bouhassira D. Diffuse analgesic effects of uniloateral repetitive transcranial magnetic stimulation (rTMS) in healthy volunteers. Pain 2009; 147: 224-232.

20 Dersh J, Polatin PB, Gatchel RJ. Chronic pain and psychopathology: research findings and theoretical considerations. Psychosom Med 2002; 64: 773-786.

21 Pincus T, Williams A. Models and measurements of depression in chronic pain. J Psychosom Res 1999; 47: 211-219.

22 Treede RD, Jensen TS, Campbell JN, Cruccu G, Dostrovsky JO, Griffin JW et al. Neuropathic pain: redefinition and a grading system for clinical and research purposes. Neurology 2008; 70: 1630-1635.

23 Price DD, McHaffie JG. Effects of heterotopic conditioning stimuli on first and second pain: A psychophysical evaluation in humans. Pain 1988; 34: 245-252.

24 Dworkin RH, O'Connor AB, Backonja M, Farrar JT, Finnerup NB, Jensen TS et al. Pharmacologic management of neuropathic pain: evidence-based recommendations. Pain 2007; 132: 237-251.

25 André-Obadia N, Mertens P, Gueguen A, Peyron R, Garcia-Larrea L. Pain relief by rTMS: differential effect of current flow but no specific action on pain subtypes. Neurology 2008; 71: 833-840.

26 Rossini PM, Burke D, Chen R, Cohen LG, Daskalakis Z, Di lorio R et al. Non-invasive electrical and magnetic stimulation of the brain, spinal cord, roots and peripheral nerves: basic principles and procedures for routine clinical and research application. An updated report from an I.F.C.N. Committee. Clin Neurophysiol 2015; 126: 1071-1107.

27 Ahdab R, Ayache SS, Brugieres P, Goujon C, Lefaucheur JP. Comparison of "standard" and "navigated" procedures of TMS coil positioning over motor, premotor and prefrontal targets in patients with chronic pain and depression. Neurophysiol Clin 2010; 40: 27-36.

28 George MS, Wassermann EM. Rapid-rate transcranial magnetic stimulation and ECT. Convuls Ther 1994; 10: 251-254.

29 Melzack R. The McGill Pain Questionnaire: major properties and scoring methods. Pain 1975; 1: 277-299.

30 Hamilton M. A rating scale for depression. J Neurol Neurosurg Psychiatry 1960; 23 : 56-62.

31 Hamilton M. The assessment of anxiety states by rating. Br J Med Psychol 1959; 32: 50-55.

32 Noguchi K, Gel YR, Brunner E, Konietschke F. nparLD: an R software package for the nonparametric analysis of longitudinal data in factorial experiments. J Stat Softw 2012; 50: $1-23$.

33 Yılmaz B, Kesikburun S, Yaşar E, Tan AK. The effect of repetitive transcranial magnetic stimulation on refractory neuropathic pain in spinal cord injury. J Spinal Cord Med 2014; 37: 397-400.

34 Apkarian AV, Bushnell MC, Treede RD, Zubieta JK. Human brain mechanisms of pain perception and regulation in health and disease. Eur J Pain 2005; 9: 463-484.

35 Baron R, Baron Y, Disbrow E, Roberts TP. Brain processing of capsaicin-induced secondary hyperalgesia: a functional MRI study. Neurology 1999; 53: 548-557.

36 Gustin SM, Wrigley PJ, Siddall PJ, Henderson LA. Brain anatomy changes associated with persistent neuropathic pain following spinal cord injury. Cereb Cortex 2010; 20: 1409-1419.

37 Stanwell P, Siddall P, Keshava N, Cocuzzo D, Ramadan S, Lin A et al. Neuro magnetic resonance spectroscopy using wavelet decomposition and statistical testing identifies biochemical changes in people with spinal cord injury and pain. Neuroimage 2010; 53: 544-552.

38 Apkarian AV, Sosa Y, Sonty S, Levy RM, Harden RN, Parrish TB et al. Chronic back pain is associated with decreased prefrontal and thalamic gray matter density. J Neurosci 2004; 24: 10410-10415.

39 Seminowicz DA, Wideman TH, Naso L, Hatami-Khoroushahi Z, Fallatah S, Ware MA et al. Effective treatment of chronic low back pain in humans reverses abnormal brain anatomy and function. J Neurosci 2011; 31: 7540-7550.

40 Obermann M, Rodriguez-Raecke R, Naegel S, Holle D, Mueller D, Yoon MS et al. Gray matter volume reduction reflects chronic pain in trigeminal neuralgia. Neuroimage 2013; 74: 352-358.

41 Yoon EJ, Kim YK, Shin HI, Lee Y, Kim SE. Cortical and white matter alterations in patients with neuropathic pain after spinal cord injury. Brain Res 2013; 2: 64-73.

42 Petrovic P, Kalso E, Petersson KM, Ingvar M. Placebo and opioid analgesia-imaging a shared neuronal network. Science 2002; 295: 1737-1740. 
43 Paus T, Castro-Alamancos MA, Petrides M. Cortico-cortical connectivity of the human mid-dorsolateral frontal cortex and its modulation by repetitive transcranial magnetic stimulation. Eur J Neurosci 2001; 14: 1405-1411.

44 Strafella AP, Paus T, Barrett J, Dagher A. Repetitive transcranial magnetic stimulation of the human prefrontal cortex induces dopamine release in the caudate nucleus. J Neurosci 2001; 21: RC157.

45 Schweinhardt P, Kalk N, Wartolowska K, Chessell I, Wordsworth P, Tracey I. Investigation into the neural correlates of emotional augmentation of clinical pain. Neuroimage 2008; 40: 759-766.

46 Avery DH, Holtzheimer PE 3rd, Fawaz W, Russo J, Neumaier J, Dunner DL et al. Transcranial magnetic stimulation reduces pain in patients with major depression: a sham-controlled study. J Nerv Ment Dis 2007; 195: 378-381.

47 Miller EK, Cohen JD. An integrative theory of prefrontal cortex function. Annu Rev Neurosci 2001; 24: 167-202.

48 Funahashi S. Neuronal mechanisms of executive control by the prefrontal cortex. Neurosci Res 2001; 39: 147-165.

49 de Oliveira RA, de Andrade DC, Mendonça M, Barros R, Luvisoto T, Myczkowski ML et al. Repetitive transcranial magnetic stimulation of the left premotor/dorsolateral prefrontal cortex does not have analgesic effect on central poststroke pain. J Pain 2014; 15: 1271-1281.

50 Boggio PS, Fregni F, Bermpohl F, Mansur CG, Rosa M, Rumi DO et al. Effect of repetitive TMS and fluoxetine on cognitive function in patients with Parkinson's disease and concurrent depression. Mov Disord 2005; 20: 1178-1184.

51 Loubinoux I, Kronenberg G, Endres M, Schumann-Bard P, Freret T, Filipkowski RK et al. Post-stroke depression: Mechanisms, translation and therapy. J Cell Mol Med 2012; 16: 1961-1969.

52 Rumi DO, Gattaz WF, Rigonatti SP, Rosa MA, Fregni F, Rosa MO et al. Transcranial magnetic stimulation accelerates the antidepressant effect of amitriptyline in severe depression: a double-blind placebo-controlled study. Biol Psychiatry 2005; 57: 162-166.

53 Fitzgerald PB, Maller JJ, Hoy KE, Thomson R, Daskalakis ZJ. Exploring the optimal site for the localization of dorsolateral prefrontal cortex in brain stimulation experiments. Brain Stimul 2009; 2: 234-237.

54 Chouinard PA, Van Der Werf YD, Leonard G, Paus T. Modulating neural networks with transcranial magnetic stimulation applied over the dorsal premotor and primary motor cortices. J Neurophysiol 2003; 90: 1071-1083. 